\title{
Fostering autonomous motivation, physical activity and cardiorespiratory fitness in rheumatoid arthritis: protocol and rationale for a randomised control trial
}

Peter C Rouse 1,2,4, Jet JCS Veldhuijzen Van Zanten ${ }^{2,4}$, George S Metsios ${ }^{3,4}$, Nikos Ntoumanis ${ }^{5}$, Chen-an Yu², Yiannis Koutedakis ${ }^{3,4}$, Sally AM Fenton ${ }^{2}$, Joanna Coast ${ }^{6}$, Hema Mistry ${ }^{7}$, George D Kitas ${ }^{4}$ and Joan L Duda ${ }^{2^{*}}$

\begin{abstract}
Background: People with rheumatoid arthritis are at greater risk of morbidity and mortality from cardiovascular disease than the general population. Sustained physical activity increases cardio-respiratory fitness and reduces cardiovascular disease risk factors. However, little is known about how we can effectively promote long-term participation in physical activity in patients with rheumatoid arthritis. The literature consistently calls for physical activity interventions, and their implementation, to be theoretically-grounded.

Methods/Design: This paper documents the protocol of a randomised control trial that investigates whether a Self-determination Theory-based intervention fosters the adoption and maintenance of physical activity (3,6 and 12 months) sufficient to provide sustained cardiovascular and personal well-being benefits in patients with rheumatoid arthritis. The cost effectiveness of the intervention will also be determined. The trial is registered as Current Controlled Trials ISRCTN04121489.

Discussion: Results from this trial will provide guidance regarding key social environmental factors that can be manipulated to support motivational processes conducive to positive health behaviour change and optimal functioning in patients with Rheumatoid Arthritis.
\end{abstract}

Keywords: Rheumatoid arthritis, Physical activity, Behaviour change, Self-determination theory, Autonomy support, Autonomous motivation

\section{Background}

Rheumatoid arthritis (RA) is the most common chronic inflammatory arthritis affecting $~ 1 \%$ of British adults [1], it causes joint pain, stiffness, swelling and can eventually lead to structural damage and physical dysfunction. Consequently, people with RA commonly experience psychological distress, particularly heightened anxiety, depression and life dissatisfaction, accompanied with feelings of reduced personal autonomy and functional independence [2-4]. In addition, RA is associated with increased morbidity and mortality, particularly from cardiovascular disease

\footnotetext{
*Correspondence: j.l.duda@bham.ac.uk

${ }^{2}$ School of Sport, Exercise and Rehabilitation Sciences, University of Birmingham, Edgbaston, Birmingham B15 2TT, UK

Full list of author information is available at the end of the article
}

(CVD) $[5,6]$, with both myocardial infarction and stroke being more prevalent than in the general population $[7,8]$. The increased risk of CVD is attributed to both classical (e.g. hypertension, dyslipidaemia) $[9,10]$ and novel or disease-related factors (e.g., inflammation) [11]. Regular physical activity (PA) of sufficient intensity increases cardio-respiratory fitness and has been repeatedly shown to reduce individual CVD risk factors as well as overall CVD risk in both healthy [12] and diseased populations such as cardiac and diabetic patients [13]. Evidence indicates that exercise interventions in patients with RA lead to reduced feelings of fatigue, improved cardio-respiratory fitness, physical function and psychological well-being, without aggravating symptoms or inducing further joint damage [14,15]. More 
recently, regular exercise was also shown to improve the cardiovascular risk profile in patients with RA [16,17].

\section{Physical activity and rheumatoid arthritis}

Even though RA patients are aware of the benefits of PA $[18,19]$, the majority of the patients are not physically active $[20,21]$. However, regular PA is a realistic and important component to a holistic treatment programme for this patient group. As mentioned above, a recent study revealed that an individualised exercise intervention induced reductions in CVD risk in patients with RA [12]. Patients who received the exercise intervention showed reductions in blood pressure, lipid ratio and inflammation, as well as increases in fitness and functional ability, compared to an advice-only control group. In other patient groups (e.g., hypertensive and overweight patients), exercise training programmes of several weeks or months in duration have enhanced insulin sensitivity [22], reduced blood pressure [23], improved lipoprotein profile and decreased body fatness [24], and may even have an anti-inflammatory effect [25]. These data clearly suggest that most CVD risk factors, which are particularly pronounced in RA patients, can be beneficially modified by increasing levels of PA engagement $[14,26]$. Still, whilst such exercise programmes have demonstrated short-term improvements in patient health [27], there is no compelling evidence for sustained participation in PA post programmes and associated long-term improvements in specific outcomes. Indeed, the possible cardiovascular benefits of exercise only persist with continued and long-term PA participation. Understanding the processes linked to adherence to individualised exercise protocols after cessation of structured programmes is therefore central to the success of PA promotion programmes which seek to bring about lasting health benefits. The mechanisms underlying maintenance of PA engagement have not been studied in RA patients [14]. Therefore, research is needed to understand how to optimally support long-term PA participation in this patient group.

This paper documents the rationale for, and protocol of a randomised control trial (RCT) that compares two 3month exercise programmes, with the primary aim of improving cardio-respiratory fitness among patients with RA. The exercise component of both programmes is the same, but one programme is supplemented by a theoreticallygrounded behaviour change intervention. Specifically, this intervention aimed to target the key motivational processes underlying PA behaviour change, with the intention of encouraging the adoption and maintenance of PA and in turn, improving cardio-respiratory fitness among RA patients.

\section{Theoretical framework}

In order to take into account key underlying processes relevant to PA adherence and optimal functioning, interventions should be theoretically based $[27,28]$. Systematic and meta-analytic reviews $[29,30]$ indicate that Self-determination Theory (SDT; [31]) holds promise for understanding the processes that lead to sustained health behaviour change and well-being. SDT is concerned with the determinants and implications of 'why' we engage in specific behaviours. Specifically, SDT focuses on the degree to which people's motivation toward engagement in activities, such as PA, emanates from the self (i.e., is self-determined) or is driven by external or internal pressures. SDT proposes that when an activity is not intrinsically motivating, behaviour is guided by a variety of extrinsic regulations which are assumed to lie on a self-determination continuum, ranging from those that are more self-determined (or autonomous) to those that are less self-determined (or controlled) [32]. Research grounded in SDT [33], has highlighted the positive influence that autonomy support (e.g., eliciting and acknowledging perspectives, supporting self-initiative, offering choice, providing relevant information and minimizing pressure and control) can have on facilitating more autonomous motivation and health behaviour change, as well as associated physical and psychological health benefits [34].

To date, within the SDT-based literature centred on PA promotion, emphasis has been placed on the degree of autonomy support offered by a variety of exercise professionals (e.g. instructors and health fitness advisors [35-38]. According to SDT, the degree to which individuals experience well-being and are more autonomous in their motivation is influenced by the extent to which their innate psychological needs to feel competent, autonomous, and connected with others (sense of relatedness) are satisfied in a particular context or activity. SDT proposes that autonomy supportive interactions with significant others (e.g., exercise instructors, behavioural change counsellors) contribute to greater satisfaction of the 3 psychological needs of competence, autonomy, and relatedness, and in turn, enhanced autonomous motivation towards PA.

SDT-based investigations focussed on health behaviour change [including recent RCTs $[39,40]$ have shown that more self-determined regulations can predict adherence to medical prescriptions [41], smoking cessation [34], weight loss [42], and glycemic control [43]. Research particularly targeting PA has revealed positive associations between autonomy support and need satisfaction and autonomous reasons for engaging in exercise $[36,44]$. For example, previous studies have shown that more autonomous motives for exercise correspond to positive outcomes such as adherence [44] and enhanced well-being [35,44-46]. A cluster randomised control trial comparing a standard exercise referral scheme with an exercise referral intervention grounded in SDT, revealed that more health 
and fitness advisor autonomy support corresponded to greater self-determined motivation over the course of the 12 week programme. Greater self-determined motivation corresponded to enhanced well-being and PA engagement at six months follow-up [35]. In addition, a longitudinal study of overweight/obese individuals involved in an Exercise on Prescription programme [36], demonstrated an increase in psychological need satisfaction over time corresponded to greater adherence to the 3-month exercise prescription and more autonomous motives for PA engagement. The latter was associated with greater wellbeing throughout the programme. With regards to RA patients, a recent study indicated that more autonomous motivation towards PA was significantly positively associated with higher levels of self-reported PA [47].

In clinical populations, PA promotion is primarily carried out via supervised, hospital based exercise programmes. However, RA patients are often inappropriately excluded from exercise programmes, which are known to reduce the risk of CVD in the general population. The present multi-component intervention is based on psychological theory as well as physiological principles of safe and appropriate exercise programmes for patients with RA [14]. In developing the intervention package, we assumed that the goals of maintained PA behaviour change and related positive health benefits necessitate the employment of different but complementary intervention strategies [27]. As such, this trial reflects an interdisciplinary collaboration between researchers with expertise in behavioural and motivation psychology, exercise physiology and rheumatology.

\section{Aims}

The present RCT aims first, to investigate whether a SDT-based psychological intervention plus exercise programme customised for patients with RA, fosters the adoption and maintenance of PA (3, 6 and 12 months) sufficient to improve $\mathrm{VO}_{2}$ max and sustain cardiovascular and personal well-being benefits in patients with RA, compared to a standard provision exercise programme customised for this particular patient group; and second, whether this intervention is cost-effective relative to an exercise programme alone. Specifically, this RCT examined the effect of the intervention on RA patients' cardiorespiratory fitness $\left(\mathrm{VO}_{2} \mathrm{max}\right)$, PA levels (self-reported and objectively assessed), CVD risk factors (e.g., blood pressure, serological markers), RA disease activity and severity, and motivational processes underlying levels of PA engagement, immediately post-programme and at 6 and 12 month follow-up (Clinical trials registration number: ISRCTN04121489).

It is hypothesised that participants randomly allocated to the multi-component intervention arm will demonstrate more autonomous motivation for PA engagement, as well as higher levels of PA and more positive wellbeing immediately post intervention, and at 6 and 12 months follow-up, compared to participants who receive the exercise programme without psychological intervention. It is also hypothesised that higher adherence to the exercise programme will be associated with an improvement in cardio-respiratory fitness, quality of life and psychological well-being, as well as experiencing reductions in markers of CVD risk (e.g., blood pressure).

\section{Methods}

Participants with RA were recruited from consecutive rheumatology outpatient clinics at the Dudley Group NHS Foundation Trust in the UK, with recruitment taking place over a 30 month period. Patients with RA, according to the ACR criteria [48], and without co-morbidities prohibiting exercise were recruited. Exclusion criteria were recent joint surgery (in preceding 6 months), fibromyalgia and co-morbidity incompatible with exercise as per ACSM guidelines. Ethical approval was obtained from the Birmingham, East, North and Solihull Research NHS Ethics Committee (protocol number 10/H1206/59).

\section{Protocol}

Patients that satisfied the inclusion criteria were approached by a member of the research team in the waiting room of rheumatology clinics and provided with information about the study. After written consent was obtained, participants were randomised into the intervention or control condition. Randomisation was completed by a third party (Cancer Clinical Trials Unit, University of Birmingham) and stratified based on gender. Participants randomised to the experimental arm were provided with an additional information sheet concerning the content of the psychological intervention (see Table 1), and then reconsented. The two stages of consent were necessary to reduce contamination between the two arms, as providing a complete description of the psychological intervention to participants in both study arms would have jeopardised the internal validity of the study.

All participants were invited to participate in a 3-month exercise programme in a local gym. The cost of the gym membership was covered to remove this known barrier to PA engagement in gym-based activities [49]. Participants completed assessments at 4 time points: baseline (before starting the exercise programme), 3 months (immediately following the completion of the exercise programme), 6 months and 12 months. Each time point consisted of securing 2 different arrays of assessments. Assessments were carried out at the Clinical Research Unit within the Dudley Group NHS Foundation Trust, 7 days apart. During the first assessment appointment (approximately 30 minutes), a fasted blood sample was taken and participants were asked to complete a questionnaire pack (See Table 2). A sub- 
Table 1 Psychological intervention content for each contact with participants

\begin{tabular}{|c|c|c|c|}
\hline Consultation & Contact & Duration & Behaviour change techniques \\
\hline \multirow[t]{6}{*}{ Baseline } & Face to face & 60 mins & - Elicit and acknowledge positive and negative experiences and emotions towards physical activity \\
\hline & & & $\begin{array}{l}\text { - Identify the patient's knowledge regarding the benefits associated with increasing physically active } \\
\text { behaviour specific to RA; gear discussions of these benefits to what is personally meaningful to the patient }\end{array}$ \\
\hline & & & - Provide additional information requested by the patient \\
\hline & & & $\begin{array}{l}\text { - Encourage reflection on the links between physically active behaviour and personally meaningful life } \\
\text { goals or events }\end{array}$ \\
\hline & & & - Decisional balance \\
\hline & & & - Patient centred goal setting \\
\hline \multirow[t]{5}{*}{1 month } & Telephone & 10 mins & - Support attempts to change behaviour \\
\hline & & & - Normalize failed attempts to be physically active \\
\hline & & & - Problem solve to formulate strategies for enhancing self-efficacy \\
\hline & & & - Elicit solutions to PA barriers \\
\hline & & & - Revisit goals \\
\hline \multirow[t]{3}{*}{2 month } & Telephone & 10 mins & - Encourage attempts made to be physically active \\
\hline & & & - Brainstorm solutions to PA barriers \\
\hline & & & - Discuss patient goals for last period of programme \\
\hline \multirow[t]{4}{*}{3 month } & Face to face & 30 mins & - Recognise the internalisation of individual's PA participation \\
\hline & & & - Have patients verbalise feelings towards physical activity \\
\hline & & & - Discuss plans to be physically active in the future \\
\hline & & & - Information regarding where it is possible to be physically active \\
\hline 5 month & Telephone & 10 mins & - Discuss successful and failed attempts to maintain PA behaviour post exercise-programme \\
\hline
\end{tabular}

sample (85\%) of participants were provided with an Actigraph accelerometer (GT3X) to be worn for 7 days. Verbal and written instructions were provided detailing how the accelerometer should be worn and a demonstration given. Participants were requested to wear the device for the next 7 days, only removing for showering/bathing.

At the second assessment appointment (taking approximately 50 minutes), an exercise tolerance test (ETT) with electrocardiography (ECG) was conducted by two trained exercise and cardiac physiologists. Assessors were blinded to the intervention arm (i.e., experimental vs. control) to which participants were randomised. Participants returned the accelerometer and completed a self-report measure of PA (i.e., The International Physical Activity Questionnaire; IPAQ) [50] along with a demographic information sheet. See Figure 1 for the study flow diagram.

\section{Physical activity intervention}

All participants, regardless of study arm, participated in a 3-month exercise programme. To avoid contamination, the two arms completed the programmes at two separate gym centres. Both gym centres were similar in terms of facilities, size, available equipment, environment. Gym instructors at both exercise centres received training in how to support patients with RA by the same exercise physiologist with extensive experience in developing exercise programmes appropriate for patients with RA. This training consisted of information about RA in general, how to develop a safe programme for this patient group, as well as the potential physical and psychological barriers RA patients may encounter while participating in an exercise programme.

Following the baseline assessment of cardio-respiratory fitness, an individualised 3-month exercise programme was developed, using all available equipment (treadmills, hand ergometers, etc) and according to the patient's preference and ability [26]. In line with the American College of Sports Medicine (ACSM) guidelines (2005) for exercise prescription, participants were asked to exercise at $60-75 \%$ of their $\mathrm{VO}_{2} \max$ (indicated by monitored heart rate) for a duration greater than 30 min during each exercise session. All exercise sessions were designed to last approximately 55mins and consisted of10mins warm-up, 30-40mins main session, and 5-10mins cooling down.

Exercisers provided one-to-one supervision to participants during their initial exercise session. This first gym session served as an induction to the exercise equipment and exercise programme. In the case that a participant required further assistance, full supervision was provided until he or she felt familiar and comfortable with the equipment and confident to exercise alone. Thereafter, patients exercised in a semi-supervised setting. Instructors 
Table 2 Secondary outcome measures for the RCT

\begin{tabular}{|c|c|}
\hline Outcome & Measure \\
\hline \multicolumn{2}{|l|}{ Physical activity related } \\
\hline Physical activity adherence & Attendance \\
\hline Physical activity (self-report) & $\begin{array}{l}\text { International Physical Activity } \\
\text { Questionnaire [50] }\end{array}$ \\
\hline Physical activity (objective) & Actigraph GT3X \\
\hline $\begin{array}{l}\text { Autonomy support } \\
\text { (fitness instructor) }\end{array}$ & Health Care Climate Questionnaire [42] \\
\hline $\begin{array}{l}\text { Autonomy support } \\
\text { (important others \& PA advisor) }\end{array}$ & $\begin{array}{l}\text { Important Other Care Climate } \\
\text { Questionnaire [51] }\end{array}$ \\
\hline Need satisfaction & $\begin{array}{l}\text { Need Satisfaction in Exercise } \\
\text { Questionnaire [52] }\end{array}$ \\
\hline Motivational regulations & $\begin{array}{l}\text { Behavioural Regulations in } \\
\text { Exercise Questionnaire-2 [53] }\end{array}$ \\
\hline \multicolumn{2}{|l|}{ Health related } \\
\hline Acute phase response & $\begin{array}{l}\text { Routine laboratory assessments } \\
\text { (i.e., C-Reactive Protein) }\end{array}$ \\
\hline Disease activity & Disease Activity Score 28 [54] \\
\hline Quality of life & EQ-5D-3L [55] \\
\hline Anxiety \& depression & $\begin{array}{l}\text { Hospital Anxiety and Depressions } \\
\text { Scale [56] }\end{array}$ \\
\hline Well-being & Subjective Vitality Scale [57] \\
\hline Fatigue & $\begin{array}{l}\text { Multidimensional Assessment of } \\
\text { Fatigue Scale [58] }\end{array}$ \\
\hline Physical function & $\begin{array}{l}\text { Stanford Health Assessment } \\
\text { Questionnaire (Kirwan \& Reeback) [59] }\end{array}$ \\
\hline Pain & McGill Pain Questionnaire [60] \\
\hline \multicolumn{2}{|l|}{ Economic costing } \\
\hline Changes in capability & ICECAP-A [61,62] \\
\hline Quality-adjusted life-years & EQ-5D-3L [55,63] \\
\hline
\end{tabular}

were available to assist if patients required any further support, or in case of emergency. In total, three exercise sessions per week were recommended to all participants; two sessions conducted in the gym environment within the designated exercise centre, and one session at home. Objective attendance records were obtained from both exercise centres. At the end of the 3-month programme, patients were left to their own devices to continue their engagement in PA.

\section{Psychological intervention}

For patients randomised to the experimental arm, the individualised 3-month exercise programme was supplemented by psychological intervention strategies grounded in SDT [64]. Strategies aimed to facilitate the adoption and maintenance of PA, whilst supporting the patients' autonomous motivation for PA engagement and psychological well-being via one-to-one consultations with a PA behavioural change counsellor [64]. The counsellor had contact with each patient at 4 time points during the 3 -

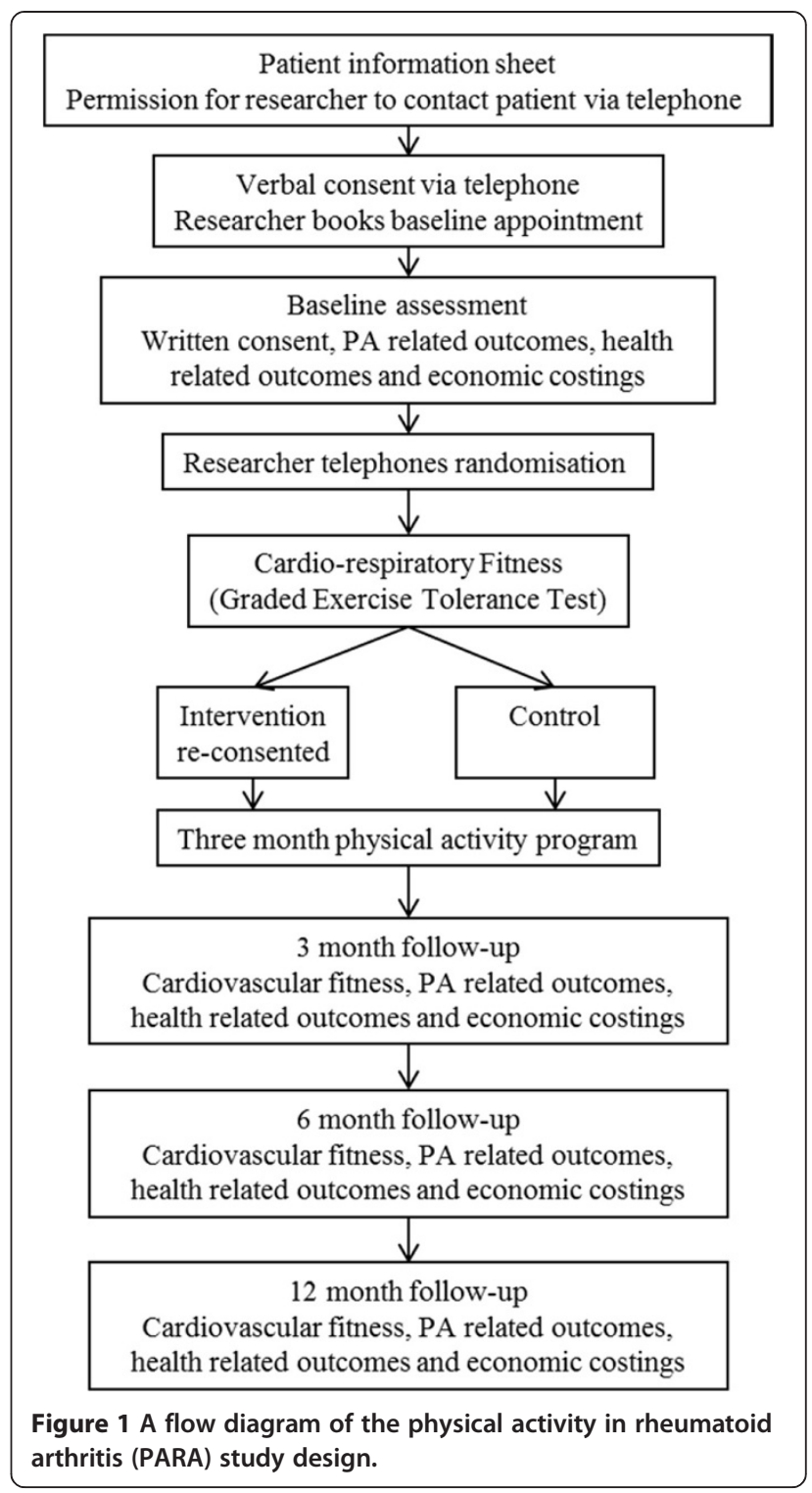

month exercise programme (i.e., a $1 \mathrm{hr}$ face to face consultation at programme induction, brief consultations via telephone at 1 and 2 months, and an approximately $30 \mathrm{~min}$ face to face consultation at 3 months). As the literature points to the need for a follow up consultation when participants are trying to maintain their PA post-exercise programme [65], a final telephone consultation took place at 5 months (i.e., 2 months following the end of the exercise intervention). This approach is in line with the recommendations of Hillsdon and colleagues [66] regarding the minimal frequency of intervention occasions, and recent work involving SDT-grounded exercise on referral consultations [28]. This protocol also parallels the SDT-based behavioural change interventions employed by Williams and colleagues [40] (smoking cessation/dietary change) and other researchers centred on PA promotion specifically [67]. 
The counsellor was trained to employ particular strategies and approaches targeting the promotion of more autonomous motivation for PA behaviour change in the participant. For example, during the initial consultation, the counsellor elicited and acknowledged positive and negative experiences and emotions towards PA, provided personally salient information regarding the benefits associated with increasing physically active behaviour specific to RA, and encouraged reflection on the links between physically active behaviour and personally meaningful life goals or events. See Table 1 for details of intervention content.

To reduce the potential that the exercise instructors who supervised the sessions might be creating an environment in the gym which is at odds to the perspective adopted within the one on one consultations, two 1.5 hours information sessions were provided. To create a need supportive exercise environment, exercise instructors at the experimental gym centre received information on the benefits of promoting autonomy (e.g,. offering choice of the types exercises included in his/her programme), providing structure to enhance feelings of competence (e.g., encouraging patients to ask question) and supporting relatedness (e.g., dedicating time and attention to the patient) during exercise sessions with RA patients. Within these sessions, the instructors were encouraged to identify current good practice and generate strategies by which they could be more need supportive when supervising gym activities.

\section{Outcomes}

\section{Primary outcome}

Cardio-respiratory fitness (CRF) was the primary outcome and was assessed using a graded exercise tolerance test, either on a treadmill (HP Cosmos Mercury, Nussdorf-Traunstein, Germany) or an exercise bike (Lode, The Netherlands), depending on the patient's ability and preference. Based on guidelines from the American Heart Association, an individualised test protocol was utilised to assess CRF [68]. The testing initiated in most cases at $2 \mathrm{mph}$ and $1 \%$ inclination. The speed increased by $0.5 \mathrm{mph}$ every 1 minute until $4.0 \mathrm{mph}$ followed, thereafter, by inclination increases every 30 seconds by $1 \%$. In all cases, the aim was to achieve exhaustion within 7-12 minutes. CRF was determined using a calibrated breath-bybreath system (Metalyzer 3B, CORTEX Biophysik GmbH, Leipzig, Germany) allowing continuous measurement of oxygen uptake $\left(\mathrm{VO}_{2}\right)$, and lung ventilation, determined once every 30 seconds. Additionally, blood pressure was monitored and heart rate and function were continuously assessed via a 12 lead electrocardiogram.

\section{Secondary outcomes CVD risk factors}

Resting blood pressure and serological risk factors, including full blood count, serum biochemistry, Erythrocyte
Segmentation Rate (ESR), high-sensitivity C-Reactive Protein (hsCRP), blood glucose, lipids and thrombotic variables were assessed using routine laboratory procedures. Internal quality controls on all analysers are carried out daily, and external quality controls fortnightly, utilising the Welsh External Quality Assurance Screen (WEQAS). On the basis of these individual CVD risk factors, the 10-year CVD event probability was calculated using the latest (2006) version of the Joint British Societies' risk calculator [69].

\section{Physical activity behaviours}

(a) Adherence to intervention: assessment of attendance at weekly exercise sessions, (b) self reported PA was be assessed using the International Physical Activity Questionnaire (IPAQ) [50]. The IPAQ measures the level of PA across four domains; leisure time PA, domestic and gardening (yard) activities, work - related PA, and transport - related PA, and (c) objective PA assessed by Actigraph accelerometers (GT3X) over a 7 day period in a sub-sample from each arm (experimental, $\mathrm{N}=50$, control, $\mathrm{N}=48$ ).

\section{Health and well being indicators}

(a) Health related quality of life was measured using the EuroQol, five dimensions, three levels (EQ-5D-3L) [55]. The EQ-5D-3L determines self-assessed problems across five items of mobility, self-care, usual activities, pain/discomfort and anxiety/depression. Each item has three levels of severity: 'no problems', 'some problems' and 'severe problems'. The resulting health states were valued using the UK tariff [63]. (b) anxiety and depression were assessed using the Hospital Anxiety and Depression Status (HADS) questionnaire [56]. The HADS consists of 14 items (7 each for anxiety and depression) rated on various four-point scoring systems (c) positive mental health was assessed via the Subjective Vitality Scale [57], (d) capability wellbeing was assessed using the ICECAP-A capability index for adults [61]. The ICECAP-A consists of five items of Attachment, Stability, Achievement, Enjoyment and Autonomy. The resulting capability states were valued using the UK tariff [62].

\section{Motivation and processes of change}

(a) Perceptions of autonomy support, perceived efficacy/ competence, autonomy, social connectedness with respect to PA engagement, and motivational regulations for exercise were determined using existing scales which were validated for use in the case of RA patients.

Perceptions of autonomy support were assessed using an adapted version of the Health Care Climate Questionnaire (HCCQ) [42]. This 15 item scale was designed to measure the degree of autonomy support (vs. controllingness) perceived to be provided by the PA counsellor and their exercise instructor (e.g., I feel my PA advisor has provided me 
with choices and options). Answers were responded to on a 7-point Likert scale ranging from 1 (strongly disagree) to 7 (strongly agree).

Perceived competence, autonomy and social connectedness were assessed via participants responses to the 18item Psychological Need Satisfaction in Exercise Scale (PNES) [52]. This measure consists of three, six item subscales, i.e., perceived competence (e.g., I am confident that I can do even the most challenging exercises), autonomy (e.g., I feel free to exercise in my own way) and relatedness (e.g., I feel close to my exercise companions who appreciate how difficult exercise can be). Answers are rated on a 6-point Likert scale ranging from 1 (false) to 6 (true).

Motivation regulations were measured using an adapted version of the Behavioural Regulation in Exercise Questionnaire (BREQ-2) [53]. This 19 item questionnaire assesses behavioural regulation in the context of exercise. Specifically, participants are asked to respond to items reflecting intrinsic motivation (e.g., I exercise because it is fun), identified regulation (e.g., I value the benefits of exercise), introjected regulation (I feel guilty when I don't exercise), external regulation (e.g., I exercise because other people say I should), and amotivation (e.g. I don't see the point of exercising), rating their agreement with each statement on a 4-point Likert scale ranging from 0 (not true for me) to 4 (very true for me).

\section{RA activity and severity}

Disease Activity was measured using the DAS28 [54]. This is a composite assessment consisting of the patient's appraisal of overall health during the last week on a visual analogue scale, a 28 joint count and the current ESR. Acute phase response (i.e., CRP) was also measured using routine laboratory assessments. Physical function was assessed using the Anglicised version of the 40-item Stanford Health Assessment Questionnaire (HAQ) [59]. Patients rated their ability (over the past week) to carry out 20 activities within eight aspects of daily living (dressing/grooming, rising, eating, walking, hygiene, reach grip and errands/tasks) on a four-point scale from 'without any difficulty' to 'unable to do'. For each aspect patients also responded whether they receive assistance from people or use specific devices. Pain was assessed using the McGill Pain Questionnaire [60]. Patients are asked to rate 11 sensory descriptors (e.g., shooting, aching, splitting) and 4 affective descriptors (e.g., tiring, sickening) on an intensity scale ranging from 0 (none) to 3 (severe). Fatigue was measured using the Multidimensional Assessment of Fatigue scale (MAF) [58]. This 16-item scale measures four dimensions of fatigue: severity, distress, degree of interference in activities of daily living, and timing. Fourteen items contain numerical rating scales and two items have multiple-choice responses. Patients will be asked to reflect on fatigue patterns for the past week.

\section{Economic analysis}

The economic evaluation took the form of two costeffectiveness analyses from the perspective of the National Health Service (NHS). In the first, costs were related to the changes in quality-adjusted life-years (QALYs) developed using information collected from EQ-5D-3L [55,63]; in the second, costs were related to changes in capability using the ICECAP-A capability index for adults [61,62]. A secondary analysis took the form of a cost-consequences analysis reporting all trial outcomes and costs from the perspective of both NHS and patients.

\section{Power calculations}

Our power calculations (nQuery Advisor v 6.0, Statistical Solutions, MA, USA) were based on the primary outcome CRF and indicated that we needed 50 participants per group. This is sufficient to detect an estimated mean difference in CRF of $2 \mathrm{ml} / \mathrm{kg} / \mathrm{min}\left(\mathrm{SD}_{\text {diff. }}=3\right)$ at 1 year between the 2 groups (power $=80 \%$; alpha $=.05$ ), allowing for $20 \%$ drop out rate. However, groups were compared on an intention to treat basis, thus, even patients that drop-out were included in the analyses.

\section{Statistical analysis strategy}

Multilevel regression analyses were used to test the effect of the intervention on our outcome measures in comparison with the standard exercise provision arm, adjusting follow up scores for baseline scores (where available) and key baseline characteristics (e.g., age/sex, disease duration). Structural equation modelling was used to test a theorybased process model examining social psychological and motivation-related antecedents and mediators of change in targeted outcomes.

\section{Discussion}

This paper details the rationale and protocol for conducting an RCT investigating the effect of participation in two exercise programmes which were customised for people with RA, exercise only versus exercise with an additional psychological intervention that aimed to enhance the adoption and maintenance of PA, autonomous motivation for PA engagement, and associated indicators of health, QOL, and psychological well being. The psychological intervention was grounded in SDT [64], a contemporary motivation theory that provides guidance regarding key social environmental factors that can be manipulated to support motivational processes conducive to positive health behaviour change and optimal functioning.

\section{Abbreviations}

PA: Physical activity; RA: Rheumatoid arthritis; CVD: Cardiovascular disease CRF: Cardio-respiratory fitness; ETT: Exercise tolerance test; SDT: Selfdetermination theory; RCT: Randomised control trial; NHS: National health service; QALYs: Quality adjusted life years; EQ-5D-3L: EuroQol, five dimensions, three levels; ICECAP-A: Investigating choice experiments capability index for adults. 


\section{Competing interests}

The authors declare that they have no competing interests.

\section{Authors' contributions}

JLD, GK, JWZ and GM conceived the study. JLD, GK, JWZ, GM, NN, PR and $C Y$ were involved with the design of the study and protocol development. XX led on the analytic strategy for determining cost effectiveness. PR managed the study and organized data collections with SF also contributing. PR conducted the psychological intervention. NN prepared the statistical plan. All authors read and approved the final manuscript.

\section{Acknowledgements}

We are grateful to the patients and exercise instructors for their engagement in this research project and to the Medical Research Council National Prevention Research Initiative for funding this work.

\section{Author details}

${ }^{1}$ Department of Health, University of Bath, Bath BA2 7AY, UK. ${ }^{2}$ School of Sport, Exercise and Rehabilitation Sciences, University of Birmingham, Edgbaston, Birmingham B15 2TT, UK. ${ }^{3}$ Institute of Sport, Faculty of Health Education and WellBeing, University of Wolverhampton, Wolverhampton W1 1LY, UK. ${ }^{4}$ Department of Rheumatology, The Dudley Group NHS Foundation Trust, Russell's hall Hospital, Dudley DY1 2HQ, UK. ${ }^{5}$ School of Psychology and Speech Pathology, Curtin University, Perth 6845, Western Australia. ${ }^{6}$ School of Health and Population Sciences, University of Birmingham, Birmingham B15 2TT, UK. ${ }^{7}$ Warwick Medical School, University of Warwick, Coventry CV4 7AL, UK.

Received: 25 November 2014 Accepted: 9 December 2014 Published: 19 December 2014

\section{References}

1. Lee DM, Weinblatt ME: Rheumatoid arthritis. Lancet 2001, 358:903-911.

2. Dickens C, McGowan L, Clark-Carter D, Creed F: Depression in rheumatoid arthritis: a systematic review of the literature with meta-analysis. Psychosom Med 2002, 64:52-60.

3. Treharne GJ, Lyons AC, Booth DA, Kitas GD: Psychological well-being across 1 year with rheumatoid arthritis: coping resources as buffers of perceived stress. Br J Health Psychol 2007, 12:323-345.

4. Treharne GJ, Kitas GD, Lyons AC, Booth DA: Well-being in rheumatoid arthritis: the effects of disease duration and psychosocial factors. $J$ Health Psychol 2005, 10:457-474

5. Kitas GD, Erb N: Tackling ischaemic heart disease in rheumatoid arthritis Rheumatology 2003, 42:607-613.

6. Kitas GD, Gabriel SE: Cardiovascular disease in rheumatoid arthritis: state of the art and future perspectives. Ann Rheum Dis 2010, 70:8-14.

7. Avina-Zubieta JA, Thomas J, Sadatsafavi M, Lehman AJ, Lacaille D: Risk of incident cardiovascular events in patients with rheumatoid arthritis: a meta-analysis of observational studies. Ann Rheum Dis 2012, 71:1524-1529.

8. Avina-Zubieta JA, Choi HK, Sadatsafavi M, Etminan M, Esdaile JM, Lacaille D: Risk of cardiovascular mortality in patients with rheumatoid arthritis: a meta-analysis of observational studies. Arthritis Rheum 2008, 59:1690-1697

9. Panoulas VF, Metsios GS, Pace AV, John H, Treharne GJ, Banks MJ, Kitas GD: Hypertension in rheumatoid arthritis. Rheumatology 2008, 47:1286-1298.

10. Toms TE, Panoulas VF, Smith JP, Douglas KMJ, Metsios GS, StavropoulosKalinoglou A, Kitas GD: Rheumatoid arthritis susceptibility genes associate with lipid levels in patients with rheumatoid arthritis. Ann Rheum Dis 2011, 70:1025-1032

11. Stevens RJ, Douglas KMJ, Saratzis AN, Kitas GD: Inflammation and atherosclerosis in rheumatoid arthritis. Exp Rev Mol Med 2005, 7:1-24

12. Scrutinio D, Bellotto F, Lagioia R, Passantino A: Physical activity for coronary heart disease: cardioprotective mechanisms and effects on prognosis. Monaldi Arch Chest Dis 2005, 64:77-87.

13. Pedersen BK, Saltin B: Evidence for prescribing exercise as therapy in chronic disease. Scand J Med Sci Sports 2006, 16:3-63.

14. Metsios GS, Stavropoulos-Kalinoglou A, van Zanten JJCS, Treharne GJ, Panoulas VF, Douglas KMJ, Koutedakis Y, Kitas GD: Rheumatoid arthritis, cardiovascular disease and physical exercise: a systematic review. Rheumatology 2008, 47:239-248.
15. Cramp F, Hewlett S, Almeida C, Kirwan JR, Choy EH, Chalder T, Pollock J, Christensen R: Non-pharmacological interventions for fatigue in rheumatoid arthritis. Cochrane Database Syst Rev 2013, 23, CD008322.

16. Stavropoulos-Kalinoglou A, Metsios GS, Veldhuijzen van Zanten J, Nightingale $P$, Kitas GD, Koutedakis Y: Individualised aerobic and resistance exercise training improves cardiorespiratory fitness and reduces cardiovascular risk in patients with rheumatoid arthritis. Ann Rheum Dis 2013, 72:1819-1825

17. Metsios GS, Stavropoulos-Kalinoglou A, Veldhuijzen van Zanten JJ, Nightingale P, Sandoo A, Dimitroulas T, Kitas GD, Koutedakis Y: Individualised exercise improves endothelial function in patients with rheumatoid arthritis. Ann Rheum Dis 2014, 73:748-751.

18. Law RJ, Markland DA, Jones JG, Maddison PJ, Thom JM: Perceptions of issues relating to exercise and joint health in rheumatoid arthritis: a UK-based questionnaire study. Musculoskelet Care 2013, 11:147-158.

19. Henchoz Y, Zufferey P, So A: Stages of change, barriers, benefits, and preferences for exercise in RA patients: a cross-sectional study. Scand J Rheumatol 2013, 42:136-145.

20. Sokka T, Hakkinen A, Kautiainen H, Maillefert JF, Toloza S, Hansen TM, Calvo-Alen J, Oding R, Liveborn M, Huisman M, Alten R, Pohl C, Cutolo M, Immonen K, Woolf A, Murphy E, Sheehy C, Quirke E, Celik S, Yazici Y, Tlustochowicz W, Kapolka D, Skakic V, Rojkovich B, Müller R, Stropuviene S, Andersone D, Drosos AA, Lazovskis J, Pincus T: Physical inactivity in patients with rheumatoid arthritis: data from twenty-one countries in a cross-sectional, international study. Arthritis Rheum 2008, 59:42-50.

21. Tierney M, Fraser A, Kennedy N: Physical activity in rheumatoid arthritis: a systematic review. J Phys Act Health 2012, 9:1036-1048.

22. Henriksen EJ: Invited review: effects of acute exercise and exercise training on insulin resistance. J Appl Physiol 2002, 93:788-796.

23. Duncan JJ, Farr JE, Upton SJ, Hagan RD, Oglesby ME, Blair SN: The effects of aerobic exercise on plasma-catecholamines and blood-pressure in patients with mild essential-hypertension. JAMA 1985, 254:2609-2613.

24. Wood PD, Stefanick ML, Williams PT, Haskell WL: The effects on plasma lipoproteins of a prudent weight-reducing diet, with or without exercise, in overweight men and women. N Engl J Med 1991, 325:461-466.

25. Edwards KM, Ziegler MG, Mills PJ: The potential anti-inflammatory benefits of improving physical fitness in hypertension. J Hypertens 2007, 25:1533-1542.

26. Stavropoulos-Kalinoglou A, Metsios GS, Veldhuijzen van Zanten JJ, Nightingale P, Kitas GD, Koutedakis Y: Individualised aerobic and resistance exercise training improves cardiorespiratory fitness and reduces cardiovascular risk in patients with rheumatoid arthritis. Ann Rheum Dis 2012, 72:1819-1825.

27. Biddle SJH, Mutrie N: Psychology of physical activity: determinants, well-being and interventions. Oxon: Routledge; 2008.

28. Michie S, Hardeman W, Fanshawe T, Prevost AT, Taylor L, Kinmonth AL Investigating theoretical explanations for behaviour change: the case study of ProActive. Psychol Health 2008, 23:25-39.

29. Teixeira PJ, Carraça EV, Markland D, Silva MN, Ryan RM: Exercise, physical activity, and self-determination theory: a systematic review. Int J Behav Nutr Phys Act 2012, 9:78.

30. Ng JYY, Ntoumanis N, Thogersen-Ntoumani C, Deci EL, Ryan RM, Duda JL, Williams GC: Self-determination theory applied to health contexts: a meta-analysis. Perspect Psychol Sci 2012, 7:325-340.

31. Deci EL, Ryan RM: Handbook of self-determination research. Rochester, NY: University of Rochester Press; 2002.

32. Pelletier LG, Fortier MS, Vallerand RJ, Briere NM: Associations among perceived autonomy support, forms of self-regulation, and persistence: a prospective study. Motiv Emot 2001, 25:279-306.

33. Deci EL, Ryan RM: Intrinsic motivation and self-determination in human behaviour. New York: Plenum Publishing Company; 1985.

34. Williams GC, Gagne M, Ryan RM, Deci EL: Facilitating autonomous motivation for smoking cessation. Health Psychol 2002, 21:40-50.

35. Duda JL, Williams GC, Ntoumanis N, Daley A, Eves FF, Mutrie N, Rouse PC, Lodhia R, Blamey RV, Jolly K: Effects of a standard provision versus an autonomy supportive exercise referral programme on physical activity, quality of life and well-being indicators: a cluster randomised controlled trial. Int J Behav Nutr Phys Act 2014, 11:10.

36. Edmunds J, Ntoumanis N, Duda JL: Adherence and well-being in overweight and obese patients referred to an exercise on prescription scheme: a self-determination theory perspective. Psychol Sport Exerc 2007, 8:722-740. 
37. Fortier M, Duda J, Guerin E, Teixeira P: Promoting physical activity: development and testing of self-determination theory-based interventions. Int J Behav Nutr Phys Act 2012, 9:20.

38. Rouse PC, Ntoumanis N, Duda JL, Jolly K, Williams GC: In the beginning: role of autonomy support on the motivation, mental health and intentions of participants entering an exercise referral scheme. Psychol Health 2011, 26:729-749.

39. Fortier MS, Sweet SN, O'Sullivan TL, Williams GC: A self-determination process model of physical activity adoption in the context of a randomized controlled trial. Psychol Sport Exerc 2007, 8:741-757.

40. Williams GC, McGregor HA, Sharp D, Levesque C, Kouides RW, Ryan RM, Deci EL: Testing a self-determination theory intervention for motivating tobacco cessation: supporting autonomy and competence in a clinical trial. Health Psychol 2006, 25:91-101.

41. Williams GC, Deci EL: The importance of supporting autonomy in medical education. Ann Int Med 1998, 129:303-308.

42. Williams GC, Grow VM, Freedman ZR, Ryan RM, Deci EL: Motivational predictors of weight loss and weight-loss maintenance. J Pers Soc Psychol 1996, 70:115-126.

43. Williams GC, McGregor HA, Zeldman A, Freedman ZR, Deci EL: Testing a self-determination theory process model for promoting glycemic control through diabetes self-management. Health Psychol 2004, 23:58-66.

44. Edmunds J, Ntoumanis N, Duda JL: Testing a self-determination theory-based teaching style in the exercise domain. Eur J Soc Psychol 2008, 38:375-388.

45. Jolly K, Lip G, Sandercock J, Greenfield S, Raftery J, Mant J, Taylor R, Lane D, Lee KW, Stevens AJ: Home-based versus hospital-based cardiac rehabilitation after myocardial infarction or revascularisation: design and rationale of the Birmingham Rehabilitation Uptake Maximisation Study (BRUM): a randomised controlled trial [ISRCTN72884263]. BMC CardiovasC Disord 2003, 3:10.

46. Munneke M, de Jong Z, Zwinderman AH, Jansen A, Ronday HK, Peter WF, Boonman DC, van den Ende CH, Vliet Vlieland TP, Hazes JM: Adherence and satisfaction of rheumatoid arthritis patients with a long-term intensive dynamic exercise program (RAPIT program). Arthritis Care Res 2003, 49:665-672.

47. Hurkmans EJ, Maes S, De Gucht V, Knittle K, Peters AJ, Ronday HK, Vlieland TP: Motivation as a determinant of physical activity in patients with rheumatoid arthritis. Arthritis Care Res 2010, 62:371-377.

48. Arnett FC, Edworthy SM, Bloch DA, McShane DJ, Fries JF, Cooper NS, Healey LA, Caplan SR, Liang MH, Luthra HS, et al: The American rheumatism association 1987 revised criteria for the classification of rheumatoid arthritis. Arthritis Rheum 1988, 31:315-324.

49. Wilcox S, Der Ananian C, Abbott J, Vrazel J, Ramsey C, Sharpe PA, Brady T: Perceived exercise barriers, enablers, and benefits among exercising and nonexercising adults with arthritis: results from a qualitative study. Arthritis Care Res 2006, 55:616-627.

50. Craig CL, Marshall AL, Sjostrom M, Bauman AE, Booth ML, Ainsworth BE, Pratt M, Ekelund U, Yngve A, Sallis JF, Oja P: International physical activity questionnaire: 12-country reliability and validity. Med Sci Sports Exerc 2003, 35:1381-1395.

51. Williams GC, Lynch MF, McGregor HA, Sharp D, Deci EL, Ryan RM: Validation of the "Important Other" climate questionnaire: assessing autonomy support for health-related change. Fam Syst Health 2006, 24:179-194.

52. Wilson PM, Rogers WT, Rodgers WM, Wild TC: The psychological need satisfaction in exercise scale. J Sport Exerc Psychol 2006, 28:231-251.

53. Markland $\mathrm{D}$, Tobin $\mathrm{V}$ : A modification to the behavioural regulation in exercise questionnaire to include an assessment of amotivation. J Sport Exerc Psychol 2004, 26:191-196.

54. Van der Heijde DM, Van't Hof MA, Van Riel PL, Theunisse LA, Lubberts EW, Van Leeuwen MA, van Rijswijk MH, van de Putte LB: Judging disease activity in clinical practice in rheumatoid arthritis: first step in the development of a disease activity score. Ann Rheum Dis 1990, 49:916-920.

55. Williams A: Euroqol - a new facility for the measurement of health-related quality-of-life. Health Policy 1990, 16:199-208.

56. Zigmond AS, Snaith RP: The hospital anxiety and depression scale. Acta Psychiatr Scand 1983, 67:361-370.

57. Ryan RM, Frederick C: On energy, personality, and health: Subjective vitality as a dynamic reflection of well-being. J Pers 1997, 65:529-565.

58. Tack BB: Fatigue in rheumatoid arthritis: conditions, strategies, and consequences. Arthritis Care Res 1990, 3:65-70
59. Kirwan JR, Reeback JS: Stanford health assessment questionnaire modified to assess disability in British patients with rheumatoid arthritis. BrJ Rheumatol 1986, 25:206-209.

60. Melzack R: The short-form McGill pain questionnaire. Pain 1987, 30:191-197.

61. Al-Janabi H, Flynn TN, Coast J: Development of a self-report measure of capability wellbeing for adults: the ICECAP-A. Qual Life Res 2012, 21:167-176.

62. Flynn TN, Huynh E, Peters TJ, Al-Janabi H, Clemens S, Moody A, Coast J: Scoring the ICECAP-A capability instrument. Estimation of a UK general population tariff. Health Econ 2013, in press.

63. Dolan P: Modeling valuations for euroqol health states. Med Care 1997, 35:1095-1108

64. Deci EL, Ryan RM: The 'What' and 'Why' of goal pursuits: human needs and the self-determination of behavior. Psychol Inq 2000, 11:227-268.

65. Hughes AR, Mutrie N, Maclntyre PD: Effect of an exercise consultation on maintenance of physical activity after completion of phase III exercisebased cardiac rehabilitation. Eur J Cardiovasc Prev Rehabil 2007, 14:114-121.

66. Hillsdon M, Foster C, Cavill N, Crombie H, Naidoo B: A review of the evidence on the effectiveness of public health interventions for increasing physical activity amongst adults: a review of reviews. London: NHS Health Development Agency; 2004.

67. Fortier MS, Hogg W, O'Sullivan TL, Blanchard C, Reid RD, Sigal RJ, Boulay P, Douchet E, Sweet S, Bisson E, Beaulac J: The physical activity counselling (PAC) randomized controlled trial: rationale, methods and interventions. Appl Physiol Nutr Metab 2007, 32:1170-1185.

68. Fletcher GF, Balady GJ, Amsterdam EA, Chaitman B, Eckel R, Fleg J, Froelicher VF, Leon AS, Piña IL, Rodney R, Simons-Morton DA, Williams MA Bazzarre T: Exercise standards for testing and training - A statement for healthcare professionals from the American Heart Association. Circulation 2001, 104:1694-1740.

69. Wilson PWF, D'Agostino RB, Levy D, Belanger AM, Silbershatz H, Kannel WB: Prediction of coronary heart disease using risk factor categories. Circulation 1998, 97:1837-1847.

\section{doi:10.1186/1471-2474-15-445}

Cite this article as: Rouse et al:: Fostering autonomous motivation, physical activity and cardiorespiratory fitness in rheumatoid arthritis: protocol and rationale for a randomised control trial. BMC Musculoskeletal Disorders 2014 15:445.

\section{Submit your next manuscript to BioMed Central and take full advantage of:}

- Convenient online submission

- Thorough peer review

- No space constraints or color figure charges

- Immediate publication on acceptance

- Inclusion in PubMed, CAS, Scopus and Google Scholar

- Research which is freely available for redistribution 